\title{
Erratum to: Numerical Equilibrium Analysis for Structured Consumer Resource Models
}

\author{
A. M. de $\operatorname{Roos}^{1}$ - O. Diekmann' ${ }^{2}$ P. Getto ${ }^{3}$. \\ M. A. Kirkilionis ${ }^{4}$
}

Published online: 16 February 2016

(C) Society for Mathematical Biology 2016

\section{Erratum to: Bull Math Biol (2010) 72:259-297 DOI 10.1007/s11538-009-9445-3}

The formula for $D_{3} x$ given below equation (A9) is wrong for discontinuous $g$, and equation (A8) is also wrong. An easy way to see this is that for large $\tau$ values it does not provide a solution for (A3) (with adapted initial condition) as it should. As a result, the expressions for $D_{2} X$, the derivatives for the survival probability and the $\Psi^{i}$ appearing in the characteristic matrix also need correction. Our implementations have been for continuous $g$ and all graphs presented are correct. The mistake is related to a mistake in Diekmann et al. (2010) that appeared around the same time. For the correct formula we refer to a recently submitted corrigendum of Diekmann et al. (2010) as well as to Breda et al. (2015). The latter also contains a derivation of the correct formula.

The online version of the original article can be found under doi:10.1007/s11538-009-9445-3.

P. Getto

phgetto@yahoo.com

1 Institute for Biodiversity and Ecosystem Dynamics (IBED), University of Amsterdam, P.O. Box 94084, 1090 GB Amsterdam, The Netherlands

2 Department of Mathematics, University of Utrecht, P.O. Box 80010, 3508 TA Utrecht, The Netherlands

3 Bolyai Institute, University of Szeged, Aradi vertanuk tere 1, 6720 Szeged, Hungary

4 Department of Mathematics, University of Warwick, CV4 7AL Coventry, UK 


\section{References}

Breda D, Getto P, Sanchez Sanz J, Vermiglio R (2015) Computing the eigenvalues of realistic Daphnia models by pseudospectral methods. SIAM J Sci Comput 37(6):A2607-A2629

Diekmann O, Gyllenberg M, (=Hans) Metz JAJ, Nakaoka S, de Roos AM (2010) Daphnia revisited: local stability and bifurcation theory for physiologically structured population models explained by way of an example. J Math Biol 61:277-318. doi:10.1007/s00285-009-0299-y 